\title{
THE DECOMPOSABILITY OF TORSION FREE MODULES OF FINITE RANK
}

\author{
BY \\ EBEN MATLIS
}

Throughout this paper $R$ will be an integral domain, not a field; $Q$ will be the quotient field of $R$; and $K$ will be the $R$-module $Q / R$. $R$ will be said to have property $D$, if every torsion-free $R$-module of finite rank is a direct sum of $R$-modules of rank one.

In $[9, \S 6]$ I attempted to give necessary and sufficient conditions for an integral domain to have property $D$. I have since discovered, however, that Lemma 6.4 of that paper is false, with the result that Theorem 6.1 is also false as stated, and needs some rather strong additional hypotheses to be resurrected. Other false lemmas based on Lemma 6.4 are Proposition 6.5 and Corollary 6.7; and Lemma 6.8 remains without a valid proof.

Compounding the confusion, I have also discovered an error in Kaplansky's proof of his theorem [4] which purported to handle the Noetherian case, and this theorem remains without a valid proof. Proposition 6.11 and Corollary 6.12 of my paper [9] were based on Kaplansky's proof, and are false as stated. Corollary 6.13 remains without a valid proof.

The purpose of this paper is to attempt to restore some order out of this chaos. In Theorem $4 \mathrm{I}$ have settled the Noetherian case. This theorem states that a Noetherian domain has property $D$ if and only if it is one of two types of Noetherian domains. However, I have not been able to find an example of a ring of type (1); i.e. a principal ideal domain $R$ with exactly two nonzero prime ideals $P_{1}, P_{2}$ and such that $R_{P_{1}}, R_{P_{2}}$ are complete discrete valuation rings. It was precisely this type of ring that Kaplansky thought that he had proved could not exist. Thus one of the remaining unsolved tasks in finding all of the kinds of integral domains that have property $D$ is either to produce an example of this type of ring, or to prove that it cannot exist.

In Theorem 3 I have shown that if $R$ is an $h$-local domain with more than one maximal ideal, then $R$ has property $D$ if and only if $R$ has exactly two maximal ideals $M_{1}, M_{2}$ and $R_{M_{1}}, R_{M_{2}}$ are maximal valuation rings. The question of whether such a ring can exist is, of course, a generalization of the previous question raised.

Theorem 2 proves that a valuation ring has property $D$ if and only if it is maximal valuation ring. The major part of this result, namely that a maximal valuation ring has property $D$, is originally due to Kaplansky [3, Theorem 12] who generalized Prüfer's result [11] for complete discrete valuation rings. Both of

Received by the editors April 4, 1967. 
these authors proved the far stronger result that for the kinds of rings they were considering a torsion-free module of countable rank is a direct sum of modules of rank one.

Theorem 1 is a partial result which states that if a local domain has property $D$, then it must be complete in the $R$-topology. However, only in the Noetherian case have I been able to find necessary and sufficient conditions for a local domain to have property $D$. As indicated above, modulo the question of existence, I have solved the problem for $h$-local domains with more than one maximal ideal. Thus in order to solve the problem for all $h$-local domains only the case of a single maximal ideal, i.e. a local domain, remains to be settled.

The rings of type (2) in Theorem 4 provide examples of local domains with property $D$. This type of ring is a complete Noetherian local domain of Krull dimension one such that every ideal can be generated by two elements. The proof that a Noetherian local domain has property $D$ if and only if it is of this type is based on an earlier result of the author [7, Corollary 3] and on a theorem of Bass [1, Theorem 1.7]. The existence of rings of type (2) proves that Theorem 6.1 of [9] is false as stated. An indication of the difficulty there will be in finding necessary and sufficient conditions for a local domain to have property $D$ is given by the fact that these conditions must be sufficiently general to include both maximal valuation rings and rings of type (2).

Theorem 5 attempts to handle the integrally closed case. In the presence of a strong additional hypothesis Theorem 5 gives necessary and sufficient conditions for an integrally closed domain to have property $D$. With these additional assumptions Theorem 6.1 of [9] has been resurrected. If it could be shown that a Prüfer ring $R$ with two maximal ideals and property $D$ must have $\operatorname{inj.} \operatorname{dim}_{\cdot_{R}} R=1$, then Theorem 5 would hold for an arbitrary integrally closed domain, and this case would be solved. This conjecture on Prüfer rings seems reasonable since it holds in the $h$-local case, but I am beginning to doubt if it is true.

For the sake of convenience, we will accumulate all of the necessary definitions in the following paragraphs.

Definitions. (1) A local ring is a ring with a single maximal ideal; the ring need not be Noetherian. Some authors refer to such a ring as a quasi-local ring.

(2) An h-local ring is a ring such that each nonzero ideal is contained in only a finite number of maximal ideals, and such that each nonzero prime ideal is contained in only a single maximal ideal. A local ring is, of course, an $h$-local ring; but so is every Noetherian integral domain of Krull dimension one.

(3) A Prüfer ring is an integral domain such that every finitely generated ideal is projective. It is easily seen that an integral domain $R$ is a Prüfer ring if and only if $R_{M}$ is a valuation ring for every maximal ideal $M$ of $R$.

(4) If $A$ is an $R$-module, we will use the notation inj. $\operatorname{dim}_{._{R}} A=1$ to signify that $A$ has injective dimension one as a module over $R$. Thus, in particular, inj. $\operatorname{dim}_{\cdot_{R}} R=1$ if and only if $K$ is an injective $R$-module. 
(5) The Krull dimension of a Noetherian domain is the maximal length of a chain of prime ideals in the domain.

(6) A module over an integral domain is called $h$-divisible if it is a homomorphic image of an injective module.

(7) A module $A$ over an integral domain $R$ is called a cotorsion module if $\operatorname{Hom}_{R}(Q, A)=0$ and $\operatorname{Ext}_{R}^{1}(Q, A)=0$.

(8) Every integral domain $R$ has a natural topology, called the $R$-topology, obtained by taking the nonzero ideals as the neighborhoods of zero. This topology is a uniform topology and so $R$ has a completion; this completion is a commutative ring. We have by [8, Theorem 5.4 and Proposition 6.4] that the following statements are equivalent:

(i) $R$ is complete in the $R$-topology.

(ii) $R \cong \operatorname{Hom}_{R}(K, K)$.

(iii) $R$ is a cotorsion $R$-module.

(9) A valuation ring is called a maximal valuation ring if $R$ is complete in the $R$-topology and if inj. $\operatorname{dim}_{\cdot R} R=1$ (see [6]).

The following two lemmas were proved in [9], but we include their statements here for the sake of the coherence of the presentation.

LEMMA 1. Let $R$ be an integral domain with property $D$, and let $S$ be a subring of $Q$ containing $R$. Then $S$ also has property $D$.

Proof. See [9, Lemma 6.2].

LeMma 2. Let $R$ be an integral domain, complete in the R-topology. Then $K \bigotimes_{R} I$ has no proper, nonzero, $h$-divisible submodules for each fractionary ideal $I$ of $R$.

Proof. See [9, Lemma 6.3].

THEOREM 1. Let $R$ be a local domain with property $D$. Then $R$ is complete in the R-topology.

Proof. Consider an exact sequence of the form

$$
0 \longrightarrow R \longrightarrow A \stackrel{p}{\longrightarrow} Q \longrightarrow 0
$$

where $A$ is an $R$-module. Since $A$ is a torsion-free $R$-module of rank two, we have by property $D$ that $A=A_{1} \oplus A_{2}$, where $A_{1}, A_{2}$ are torsion-free $R$-modules of rank one. Tensoring exact sequence $\left({ }^{*}\right)$ with $K$ we obtain the isomorphism:

$$
K \cong\left(K \otimes_{R} A_{1}\right) \oplus\left(K \otimes_{R} A_{2}\right) .
$$

But $K$ is an indecomposable $R$-module by [9, Corollary 4.2]. Therefore, we can assume that $K \otimes_{R} A_{2}=0$. This shows that $A_{2} \cong Q$. Let $f$ be the restriction of $p$ to $A_{2}$. Now $f$ is nonzero; for if $f=0$, then $A_{2} \subset \operatorname{Ker} p=R$, which is impossible, since no submodule of $R$ is isomorphic to $Q$. Thus $f\left(A_{2}\right)$ is a nonzero divisible submodule 
of $Q$, and hence $f\left(A_{2}\right)=Q$. Therefore, we have $A=R+A_{2}$. Since a nonzero homomorphism of one torsion-free $R$-module of rank one into another of the same kind is a monomorphism, we have $A_{2} \cap \operatorname{Ker} p=0$; i.e. $A_{2} \cap R=0$. Hence $A=R \oplus A_{2}$, and exact sequence $\left(^{*}\right)$ is split. Since exact sequence $\left(^{*}\right)$ was an arbitrary representative of an element of $\operatorname{Ext}_{R}^{1}(Q, R)$, we have that $\operatorname{Ext}_{R}^{1}(Q, R)=0$. Thus by $[8$, Corollary 6.11] $R$ is complete in the $R$-topology.

LEMMA 3. Let $R$ be a domain, complete in the $R$-topology. Then $R$ is a maximal valuation ring if and only if inj. $\operatorname{dim}_{\cdot_{R}} I=1$ for every nonzero ideal $I$ of $R$.

Proof. If $R$ is a maximal valuation ring, then $\operatorname{inj.} \operatorname{dim}_{\cdot_{R}} I=1$ for every nonzero ideal $I$ of $R$ by [6, Theorem 4].

Conversely, assume that this condition holds. Let $a$ and $b$ be two nonzero elements of $R$, and let $I=R a \cap R b$. Since inj. $\operatorname{dim}_{\cdot R} I=1$, we have that $Q / I$ is an injective $R$-module. By Lemma $2, Q / I$ is an indecomposable, injective $R$-module.

Let $x=1+I$ in $Q / I$. Since the annihilator of $x$ is $I$, we have by [5, Theorem 2.4] that $I$ is an irreducible ideal; i.e. $I$ is not the intersection of two properly larger ideals. Thus either $I=R a$ or $I=R b$; and so either $R a \subset R b$ or $R b \subset R a$. Therefore, we have shown that $R$ is a valuation ring. Since inj. $\operatorname{dim}_{\cdot_{R}} R=1$ and $R$ is complete in the $R$-topology, $R$ is a maximal valuation ring.

THEOREM 2. Let $R$ be a valuation ring. Then $R$ is a maximal valuation ring if and only if $R$ has property $D$.

Proof. It is Kaplansky's contribution that a maximal valuation ring has property $D$ [3, Theorem 12]. Conversely, assume that $R$ has property $D$. Then $R$ is complete in the $R$-topology by Theorem 1 .

Let $I$ and $J$ be nonzero ideals of $R$, and consider an exact sequence of the form

$$
0 \longrightarrow I \longrightarrow A \stackrel{p}{\longrightarrow} J \longrightarrow 0
$$

where $A$ is an $R$-module. Since $A$ is a torsion-free $R$-module of rank two, we have by property $D$ that $A=A_{1} \oplus A_{2}$, where $A_{1}, A_{2}$ are torsion-free $R$-modules of rank one. Since $R$ is a valuation ring, the $R$-submodules of $J$ are linearly ordered. Thus we can assume that $p\left(A_{1}\right) \subset p\left(A_{2}\right)$. But $J=p\left(A_{1}\right)+p\left(A_{2}\right)$, and so $J=p\left(A_{2}\right)$. It follows that $A=I+A_{2}$. Since an $R$-homorphism of one torsion-free $R$-module of rank one into another of the same kind is either zero or a monomorphism, we have $A_{2} \cap \operatorname{Ker} p=0$; i.e. $A_{2} \cap I=0$. Thus $A=I \oplus A_{2}$, and so exact sequence ( $\left(^{*}\right)$ is split. Since exact sequence $\left(^{*}\right)$ was an arbitrary representative of an element of $\operatorname{Ext}_{R}^{1}(J, I)$, we have that $\operatorname{Ext}_{R}^{1}(J, I)=0$. $\operatorname{But}_{\operatorname{Ext}_{R}^{2}}(R / J, I) \cong \operatorname{Ext}_{R}^{1}(J, I)$, and so $\operatorname{Ext}_{R}^{2}(R / J, I)=0$. Since $J$ was an arbitrary ideal of $R$, this shows that inj. $\operatorname{dim}_{\cdot} I=1$ for every nonzero ideal $I$ of $R$. Therefore $R$ is a maximal valuation ring by Lemma 3 .

The following lemma was proved in [9]. 
LemMA 4. Let $R$ be an h-local domain with more than two maximal ideals. Then $R$ has an indecomposable, torsion-free $R$-module of rank two.

Proof. See [9, Proposition 6.9].

Lemma 5. Let $R$ be an h-local domain with more than one maximal ideal. If $R$ has property $D$, then inj. $\operatorname{dim}_{\cdot_{R}} I=1$ for every nonzero ideal $I$ of $R$.

Proof. Assume that $R$ has property $D$, and let $I$ be a nonzero ideal of $R$ such that

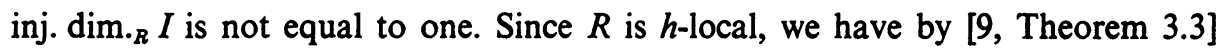
that

$$
\text { inj. } \operatorname{dim}_{\cdot_{R}} I=\text { sup. inj. } \operatorname{dim}_{R_{M}} I_{M}
$$

where $M$ ranges over all of the maximal ideals of $R$. Thus there exists a maximal ideal $M$ of $R$ such that inj. $\operatorname{dim}_{R_{M}} I_{M}>1$. It follows immediately from [2, Proposition 4.1.3] that we have inj. $\operatorname{dim}_{\cdot_{R}} I_{M}>1$ also. Therefore, there exists a nonzero ideal $J$ of $R$ such that $\operatorname{Ext}_{R}^{2}\left(R / J, I_{M}\right) \neq 0$. Since $\operatorname{Ext}_{R}^{2}\left(R / J, I_{M} \cong \operatorname{Ext}_{R}^{1}\left(J, I_{M}\right)\right.$, we have $\operatorname{Ext}_{R}^{1}\left(J, I_{M}\right) \neq 0$.

Thus we have an exact sequence:

$$
0 \longrightarrow I_{M} \longrightarrow A \stackrel{f}{\longrightarrow} J \longrightarrow 0
$$

(where $A$ is a torsion-free $R$-module of rank two) which is not a split exact sequence. Since $R$ has property $D, A=A_{1} \oplus A_{2}$, where $A_{1}, A_{2}$ are torsion-free $R$-modules of rank one. We can assume that the projection $g$ of $I_{M}$ into $A_{1}$ is not zero. Since an $R$-homomorphism of one torsion-free $R$-module of rank one into another of the same kind is either zero or a monomorphism, we have that $g$ is a monomorphism.

Thus we have an exact sequence:

$$
0 \longrightarrow I_{M} \stackrel{g}{\longrightarrow} A_{1} \longrightarrow B \longrightarrow 0
$$

where $B$ is a torsion $R$-module. From this exact sequence we derive the exact sequence:

$$
\operatorname{Hom}_{R}(B, J) \rightarrow \operatorname{Hom}_{R}\left(A_{1}, J\right) \rightarrow \operatorname{Hom}_{R}\left(I_{M}, J\right) .
$$

Since $B$ is a torsion $R$-module, we have that $\operatorname{Hom}_{R}(B, J)=0$. Now $R_{M}$ has property $D$ by Lemma 1 , and so $R_{M}$ is complete in the $R_{M}$-topology by Theorem 1 . Therefore $R_{M}$ is a cotorsion $R_{M}$-module by [8, Corollary 6.11]. It follows from [8, Lemma 1.1] that $I_{M}$ is also a cotorsion $R_{M}$-module, and thus $\operatorname{Ext}_{R_{M}}^{1}\left(Q, I_{M}\right)=0$. $\operatorname{Since}_{\operatorname{Ext}_{R}^{1}}^{1}\left(Q, I_{M}\right)$ $\cong \operatorname{Ext}_{R_{M}}^{1}\left(Q, I_{M}\right)$ by [2, Proposition 4. 1.3], we have that $I_{M}$ is also a cotorsion $R$ module. By [8, Theorem 8.5] a complete $h$-local domain has to be a local domain. Therefore, since $R$ is an $h$-local domain with more than one maximal ideal, $R$ is not complete in the $R$-topology. Thus by [8, Corollary 6.11] $R$ is not a cotorsion $R$ module. But then by [8, Proposition 5.11] an $R$-homomorphism from a cotorsion $R$-module into $R$ must be zero. Therefore, we have $\operatorname{Hom}_{R}\left(I_{M}, J\right)=0$. Since the end modules of exact sequence (3) are zero, we must have $\operatorname{Hom}_{R}\left(A_{1}, J\right)=0$ also. 
Returning to exact sequence (1) we see that $\operatorname{Hom}_{R}\left(A_{1}, J\right)=0$ implies that $f\left(A_{1}\right)=0$. Thus $A_{1} \subset \operatorname{Ker} f=I_{M}$. It follows that $f\left(A_{2}\right)=J$, and so $I_{M}+A_{2}=A$. Since $A_{2}$ and $J$ are torsion-free $R$-modules of rank one, an $R$-homomorphism of $A_{2}$ into $J$ is either zero or a monomorphism. Therefore, $A_{2} \cap \operatorname{Ker} f=0$; i.e. $A_{2} \cap I_{M}=0$. Hence we have $A=I_{M} \oplus A_{2}$. This shows that exact sequence (1) is split, in contradiction to our choice of exact sequence (1) as a nonsplit sequence. This contradiction proves that inj. $\operatorname{dim}_{\cdot R} I=1$ for every nonzero ideal $I$ of $R$.

THEOREM 3. Let $R$ be an h-local domain with more than one maximal ideal. Then $R$ has property $D$ if and only if $R$ has only two maximal ideals $M_{1}, M_{2}$ and $R_{M_{1}}, R_{M_{2}}$ are maximal valuation rings. In this case $R$ is a Prüfer ring.

Proof. It was established in [9, Lemma 6.10] that if the conditions are satisfied, then $R$ has property $D$. Hence let us assume that $R$ has property $D$. By Lemma 4, $R$ has only two maximal ideals $M_{1}$ and $M_{2}$.

Let $J$ be a nonzero ideal of $R_{M_{1}}$, and let $I=J \cap R$. By [9, Theorem 3.3] we have that

$$
\text { inj. } \operatorname{dim}_{\cdot R} I=\text { sup. inj. } \operatorname{dim}_{R_{M_{i}}} I_{M_{i}}
$$

for $i=1,2$. By Lemma 5 , inj. $\operatorname{dim}_{\cdot R} I=1$, and thus inj. $\operatorname{dim}_{R_{M_{1}}} I_{M_{1}}=1$ also. Since $J=I_{M_{1}}$, we have inj. $\operatorname{dim}_{R_{M_{1}}} J=1$. Thus every nonzero ideal of $R_{M_{1}}$ has injective dimension one over $R_{M_{1}}$.

By Lemma $1, R_{M_{1}}$ has property $D$, and thus by Theorem $1, R_{M_{1}}$ is complete in the $R_{M_{1}}$-topology. Therefore, by Lemma $3, R_{M_{1}}$ is a maximal valuation ring. Similarly, $R_{M_{2}}$ is a maximal valuation ring.

It is well known that if $R_{M}$ is a valuation ring for every maximal ideal $M$ of $R$, then $R$ is a Prüfer ring.

THEOREM 4. Let $R$ be a Noetherian integral domain. Then $R$ has property $D$ if and only if $R$ is one of the following two types of rings:

(1) $R$ is a principal ideal domain with only two distinct, nonzero, prime ideals $P_{1}, P_{2}$ and $R_{P_{1}}, R_{P_{2}}$ are complete discrete valuation rings.

(2) $R$ is a complete Noetherian local domain of Krull dimension one, and every ideal of $R$ can be generated by two elements.

Proof. Assume that $R$ is a ring of type (1). Since a complete discrete valuation ring is a maximal valuation ring, $R$ has property $D$ by Theorem 3. Assume that $R$ is a ring of type (2). Then the integral closure of $R$ is a finitely generated $R$-module (see [7, Theorem 4]). Hence by Bass' theorem [1, Theorem 1.7], every finitely generated torsion-free $R$-module is a direct sum of $R$-modules of rank one. But by [7, Corollary 3] every torsion-free $R$-module of finite rank is the direct sum of a divisible $R$-module and a finitely generated $R$-module. Thus $R$ has property $D$.

Conversely, assume that $R$ has property $D$. Suppose that $R$ has three distinct prime ideals $P_{1}, P_{2}, P_{3}$ of rank one. Let $S=R-\left(P_{1} \cup P_{2} \cup P_{3}\right)$; then $R_{s}$ has exactly three maximal ideals and is an $h$-local domain. By Lemma $1, R_{s}$ has property $D$. 
But this contradicts Lemma 4. Hence $R$ has at most two distinct prime ideals of rank one. In this case it is well known that $R$ must have Krull dimension one, and that $R$ has at most two maximal ideals.

A Noetherian domain of Krull dimension one is an $h$-local ring. Thus we have two cases to consider.

Case I. $R$ has exactly two distinct maximal ideals $M_{1}, M_{2}$.

By Theorem $3, R$ is a Prüfer ring. A Noetherian Prufer ring with only two maximal ideals is a principal ideal domain. By Theorem 3, $R_{M_{1}}$ and $R_{M_{2}}$ are maximal valuation rings. But a Noetherian maximal valuation ring is a complete discrete valuation ring. Thus $R$ is a ring of type (1).

Case II. $R$ is a Noetherian local domain.

By Theorem 1, $R$ is complete in the $R$-topology. Let $M$ be the maximal ideal of $R$. Since $R$ has Krull dimension one, the $R$-topology and the $M$-adic topology coincide. Therefore the integral closure of $R$ is a finitely generated $R$-module (see [7, Theorem 4]). Thus we can apply Bass' theorem [1, Theorem 1.7] to conclude that every ideal of $R$ can be generated by two elements, and so $R$ is a ring of type (2).

LEMMA 6. Let $R$ be a Prüfer ring with at most two maximal ideals; and suppose that $R_{M}$ is a maximal valuation ring for each maximal ideal $M$ of $R$. Then the following statements are equivalent:

(1) $R$ is an h-local ring.

(2) inj. $\operatorname{dim}_{\cdot R} R=1$.

Proof. Assume that $R$ is an $h$-local ring, and let $M_{1}$ and $M_{2}$ be the maximal ideals of $R$ (it does not matter in this case if $M_{1}=M_{2}$ ). Since $R_{M_{1}}$ is a maximal valuation ring, we have inj. $\operatorname{dim}_{R_{M_{i}}} R_{M_{i}}=1$ for $i=1,2$. By [9, Theorem 3.3] we have that

$$
\text { inj. } \operatorname{dim}_{R_{R}} R=\sup . \operatorname{inj} . \operatorname{dim} \cdot_{M_{i}} R_{M_{i}} .
$$

Thus inj. $\operatorname{dim}_{._{R}} R=1$.

Conversely, assume that inj. $\operatorname{dim}_{\cdot} R=1$. Suppose that $R$ is not an $h$-local ring. Then, in particular, $R$ has exactly two distinct maximal ideals $M_{1}$ and $M_{2}$. Let $S=R_{M_{1}}+R_{M_{2}}$ in $Q$. Since $R=R_{M_{1}} \cap R_{M_{2}}$, we have an exact sequence of $R$ modules:

$$
0 \rightarrow R \rightarrow R_{M_{1}} \oplus R_{M_{2}} \rightarrow S \rightarrow 0 .
$$

Because $R_{M_{1}}$ is complete in the $R_{M_{1}}$-topology, $R_{M_{1}}$ is a cotorsion $R_{M_{1}}$-module by [8, Corollary 6.11]. It follows from [2, Proposition 4.1.3] that $R_{M_{1}}$ is also a cotorsion $R$-module. Similarly $R_{M_{2}}$ is a cotorsion $R$-module; and hence so is $R_{M_{1}} \oplus R_{M_{2}}$.

Let $H$ be the completion of $R$ in the $R$-topology. Since $S$ is a torsion-free $R$ module, we have by [8, Proposition 2.8] that the above monomorphism $R \rightarrow R_{M_{1}}$ $\oplus R_{M_{2}}$ can be extended to a monomorphism $H \rightarrow R_{M_{1}} \oplus R_{M_{2}}$. Thus we have an induced monomorphism $H / R \rightarrow S$. Now $H / R$ is torsion-free and divisible by 
[8, Proposition 5.2] and $S$ is a torsion-free $R$-module of rank one, and so this monomorphism is either zero or an isomorphism. If it is an isomorphism, then $H \cong R_{M_{1}} \oplus R_{M_{2}}$, and $R$ is an $h$-local ring by [8, Theorem 8.5]. This contradicts our assumption on $R$, and thus $H / R=0$; i.e. $R$ is complete in the $R$-topology.

Because $R$ is not an $h$-local ring there exists a nonzero prime ideal $P$ of $R$ such that $P \subset M_{1} \cap M_{2}$. By Zorn's Lemma we can assume that $P$ is maximal among prime ideals contained in $M_{1} \cap M_{2}$. Let $V=R_{P}$; then $R_{M_{1}} \subset V$, and so $V$ is a valuation ring. Since $P V$ is the maximal ideal of $V$, we have that $P V \subset R_{M_{1}}$. Similarly $P V \subset R_{M_{2}}$, and thus $P V \subset R_{M_{1}} \cap R_{M_{2}}=R$. But $P=P V \cap R$, and so $P=P V$.

Let $\bar{R}$ be the integral domain $R / P, \bar{Q}$ the quotient field of $\bar{R}$, and $\bar{K}=\bar{Q} / \bar{R}$. Then $\bar{Q}=V|P V=V| P$, and so $\bar{K} \cong V \mid R$. We will identify $\bar{K}$ with $V / R$ whenever necessary. In particular, we have an exact sequence of $R$-modules:

$$
0 \rightarrow \bar{K} \rightarrow K \rightarrow Q / V \rightarrow 0 .
$$

Since inj. $\operatorname{dim}_{\cdot R} R=1, K$ is an injective $R$-module. Therefore, from the exact sequence (i) we derive an exact sequence:

$$
\operatorname{Hom}_{R}(K, K) \rightarrow \operatorname{Hom}_{R}(\bar{K}, K) \rightarrow 0 .
$$

We have an isomorphism $\operatorname{Hom}_{R}(\bar{K}, K) \cong \operatorname{Hom}_{\bar{R}}(\bar{K}, \bar{K})$. For let $f \in \operatorname{Hom}_{R}(\bar{K}, K)$ and let $x \in \bar{K}=V / R$. Then $x=r / s+R$, where $r \in R$ and $s \in R-P$. Thus $s f(x)=0$, which shows that $f(x) \in V \mid R=\bar{K}$. Hence we have $\operatorname{Hom}_{R}(\bar{K}, K) \cong \operatorname{Hom}_{R}(\bar{K}, \bar{K})$. Since $P \bar{K}=0$, it follows that $\operatorname{Hom}_{R}(\bar{K}, \bar{K}) \cong \operatorname{Hom}_{\bar{R}}(\bar{K}, \bar{K})$.

Because of this isomorphism we can write exact sequence (ii) in the form:

$$
\operatorname{Hom}_{R}(K, K) \rightarrow \operatorname{Hom}_{\bar{R}}(\bar{K}, \bar{K}) \rightarrow 0 \text {. }
$$

The fact that $R$ is complete in the $R$-topology gives us an isomorphism $R \cong \operatorname{Hom}_{R}(K, K)$, and we make this identification in (iii). Thus (iii) induces an exact sequence of $\bar{R}$-modules:

$$
\bar{R} \rightarrow \operatorname{Hom}_{\bar{R}}(\bar{K}, \bar{K}) \rightarrow 0 .
$$

Since $\bar{K}$ is a divisible $\bar{R}$-module, $\operatorname{Hom}_{\bar{R}}(\bar{K}, \bar{K})$ is torsion-free as an $\bar{R}$-module. Therefore (iv) is really an isomorphism: $\bar{R} \cong \operatorname{Hom}_{\bar{R}}(\bar{K}, \bar{K})$, which shows that $\bar{R}$ is complete in the $\bar{R}$-topology by [8, Proposition 6.4].

Now $\bar{R}$ has exactly two maximal ideals, and $P$ was chosen to be maximal among the prime ideals contained in $M_{1} \cap M_{2}$. Therefore, $\bar{R}$ is an $h$-local domain. By [8, Theorem 8.5] a complete $h$-local domain is in fact a local ring. This contradiction proves that $R$ must have been an $h$-local domain to begin with.

THEOREM 5. Let $\dot{R}$ be an integrally closed domain such that if $S$ is any Prüfer ring between $R$ and $Q$, then inj. $\operatorname{dim}_{\cdot R} S=1$. Then $R$ has property $D$ if and only if the following conditions are satisfied:

(1) $R$ is an h-local domain with at most two maximal ideals.

(2) $R_{M}$ is a maximal valuation ring for each of the maximal ideals $M$ of $R$. In this case $R$ is a Prüfer ring. 
Proof. If the two conditions are satisfied, then $R$ has property $D$ by either Theorem 2 or Theorem 3. Conversely, assume that $R$ has property $D$.

Case I. Suppose the collection of valuation rings between $R$ and $Q$ is linearly ordered. In this case the intersection of the valuation rings between $R$ and $Q$ is again a valuation ring. Since $R$ is integrally closed, $R$ is equal to this intersection. Thus $R$ is a valuation ring. By Theorem $2, R$ is a maximal valuation ring.

Case II. Suppose there exist two valuation rings $W_{1}$ and $W_{2}$ between $R$ and $Q$ such that $W_{1} \notin W_{2}$ and $W_{2} \notin W_{1}$. By Zorn's Lemma there exists a valuation ring $V_{1}$ such that $R \subset V_{1} \subset W_{1}$ and such that $V_{1}$ is minimal in the collection of valuation rings between $R$ and $W_{1}$. Similarly, there exists a valuation ring $V_{2}$ such that $R \subset V_{2} \subset W_{2}$ and such that $V_{2}$ is minimal in the collection of valuation rings between $R$ and $W_{2}$.

We will prove that $R=V_{1} \cap V_{2}$. Let $V$ be any valuation ring between $R$ and $Q$, and let $S=V \cap V_{1} \cap V_{2}$. Suppose that there are no containment relations between any two of the valuation rings $V, V_{1}, V_{2}$. By the independence of valuations [10, Theorem 11.11], $S$ has exactly three maximal ideals. Since $S$ has property $D$ by Lemma $1, S$ is not an $h$-local ring by Lemma 4 . Hence there exists a nonzero prime ideal contained in the intersection of two of the maximal ideals of $S$. Thus at least one of $S_{1}=V \cap V_{1}, S_{2}=V \cap V_{2}$, and $S_{3}=V_{1} \cap V_{2}$ is not an $h$-local ring.

By [10, Theorem 11.11] each of $S_{1}, S_{2}$, and $S_{3}$ is a Prüfer ring with exactly two maximal ideals, and the localizations with respect to these maximal ideals give us back $V, V_{1}$, and $V_{2}$. By Theorem 2 each of these valuation rings is a maximal valuation ring. The hypothesis of the theorem guarantees that each of $S_{1}, S_{2}$, and $S_{3}$ has injective dimension one as a module over itself. Hence we can apply Lemma 6 to conclude that each of $S_{1}, S_{2}$, and $S_{3}$ is an $h$-local ring. This contradiction shows that there is a containment relationship between two of the rings $V, V_{1}$, and $V_{2}$. The minimality of $V_{1}$ and $V_{2}$ forces this relationship to be either $V_{1} \subset V$, or $V_{2} \subset V$, or $V_{1}=V_{2}$.

Suppose that $V_{1}=V_{2}$. Let $m\left(W_{1}\right)$ and $m\left(W_{2}\right)$ be the maximal ideals of $W_{1}$ and $W_{2}$, respectively. Since $V_{1} \subset W_{1}$ and $V_{2} \subset W_{2}$, we have $m\left(W_{1}\right) \subset V_{1}$ and $m\left(W_{2}\right) \subset V_{2}$. The ideals of $V_{1}$ are linearly ordered, and $V_{1}=V_{2}$. Thus either $m\left(W_{1}\right) \subset m\left(W_{2}\right)$, or $m\left(W_{2}\right) \subset m\left(W_{1}\right)$. But then either $W_{2} \subset W_{1}$, or $W_{1} \subset W_{2}$. This contradicts our assumption that $W_{1}$ and $W_{2}$ have no containment relationships, and so we can not have $V_{1}=V_{2}$.

Thus we must have either $V_{1} \subset V$ or $V_{2} \subset V$. Since $V$ was an arbitrary valuation ring in $Q$ containing $R$, it follows that $V_{1} \cap V_{2}$ is the intersection of all of the valuation rings containing $R$. Since $R$ is integrally closed, we have $R=V_{1} \cap V_{2}$.

As we have already seen, $R=V_{1} \cap V_{2}$ is an $h$-local ring with exactly two maximal ideals, and the localizations $V_{1}$ and $V_{2}$ with respect to these maximal ideals are maximal valuation rings.

Added in proof. It has been pointed out to me by $\mathrm{D}$. Lazard that the question about the existence of rings of type (1) raised in the introduction was answered by 
F. K. Schmidt in Mehrfach perfekte Körper, Math. Ann. 108 (1933), 1-25. He proved that a field cannot be complete for two distinct discrete valuations. Mrs. Barbara Osofsky has also communicated to me a short direct proof that rings of type (1) cannot exist.

Thus Theorem 4 of this paper should read as follows:

Let $R$ be a Noetherian integral domain. Then $R$ has property $D$ if and only if $R$ is a complete local domain of Krull dimension one such that every ideal of $R$ can be generated by two elements.

\section{REFERENCES}

1. H. Bass, Torsion-free and projective modules, Trans. Amer. Math. Soc. 102 (1962), 319-327.

2. H. Cartan and S. Eilenberg, Homological algebra, Princeton Univ. Press, Princeton, N. J., 1956.

3. I. Kaplansky, Modules over Dedekind rings and valuation rings, Trans. Amer. Math. Soc. 72 (1952), 327-340.

4. - Decomposability of modules, Proc. Amer. Math. Soc. 13 (1962), 532-535.

5. E. Matlis, Injective modules over Noetherian rings, Pacific J. Math. 8 (1958), 511-528.

6. - Injective modules over Prüfer rings, Nagoya Math. J. 15 (1959), 57-69.

7. - Some properties of Noetherian domains of dimension one, Canad. J. Math. 13 (1961), 569-586.

8. - Cotorsion modules, Mem. Amer. Math. Soc. No. 49, 1964.

9. - Decomposable modules, Trans. Amer. Math. Soc. 125 (1966), 147-179.

10. M. Nagata, Local rings, Interscience, New York, 1962.

11. H. Prüfer, Theorie der Abelschen Gruppen, II. Ideale Gruppen, Math. Z. 22 (1925), 222-249.

NORTHWESTERN UNIVERSITY,

Evanston, IlLINOIS 